\title{
L'IMAGE DE LA GRÈCE DANS L'ALLEMAGNE ROMANTIQUE ET DES LUMIÈRES (SCHILLER, HEGEL ET HÖLDERLIN)
}

À la mémoire de Jacques Taminiaux

\begin{abstract}
A real fascination with Greece arose in Germany at the end of the $18^{\text {th }}$ century with the works of Winckelmann, the founder of scientific archeology. The subject of this text is the German nostalgia for Greece which developed at that time in the circle of the first romanticists and among the thinkers of German idealism. The emphasis will be put on three of the most important figures of German poetry and philosophy of this period: Schiller, Hegel and Hölderlin. But it will be shown that it is only with Hölderlin that for this idealized image of Greece a new image of Greece as profoundly divided between Occident and Orient was substituted.
\end{abstract}

Keywords: Grecomania, nostalgia, romanticism, antiquity, modernity, philosophy of history.

Dans le dernier chapitre du Crépuscule des Idoles, intitulé Ce que je dois aux Anciens, Nietzsche écrit: Il n'y a rien à apprendre des Grecs, leur génie nous est trop étranger, il est également trop fluide, pour avoir un effet impératif et «classique». ${ }^{1}$ Près de quatre-vingt dix ans auparavant, en 1801, Hölderlin, tout en affirmant à son ami Böhlendorff que les Grecs nous sont indispensables, l'avertissait aussi du danger de tirer les règles de notre art de la seule perfection grecque $^{2}$ et déclarait peu après à l'éditeur de ses traductions de Sophocle que

- Adresse pour correspondance: Route de Berzeme, La Blache, 07580 St Pons, France. Email: fr.dastur@gmail.com.

${ }^{1}$ F. Nietzsche, Crépuscules des Idoles, p. 167.

${ }^{2}$ Lettre du 4 décembre 1801 in: F. Hölderlin, Euvres, pp. 1003-1004. 
l'art grec nous est étranger ${ }^{1}$. Fremd, étranger, c'est le même terme utilisé à la fois par Nietzsche et par Hölderlin pour caractériser ce qui est grec et c'est aussi leurs deux noms que Heidegger associera, dans son cours de 1936 sur La volonté de puissance en tant qu'art, pour souligner qu'ils ont été les seuls à dégager le classique des malentendus classicistes et humanistiques en s'opposant aux positions de Winckelmann et de Goethe et en redécouvrant, derrière l'idéal d'un naturel compatible avec la raison que prône le classicisme, ce caractère propre à la nature que les Grecs de la grande époque nommaient le deinon et le deinotaton, l'effroyable ${ }^{2}$. Qu'il y ait quelque chose de profondément étranger, d'obscur et d'inquiétant dans la Grèce antique qui ne permette plus simplement de parler, selon la formule classique, de griechische Heiterkeit, de sérénité grecque ${ }^{3}$, c'est bien ce que Nietzsche a entrepris de montrer dès $L a$ naissance de la tragédie, en faisant apparaître, sous la belle apparence et la mesure caractérisant la civilisation apollinienne, la nature barbare et titanesque de son fondement dionysiaque. Nietzsche donne ainsi une importance fondamentale à ce dieu oriental qu'est Dionysos pour la définition de ce qui constitue le propre du grec.

Or c'est ce dualisme de l'apollinien et du dionysiaque qui divise profondément la Grèce antique et qui l'empêche d'accéder à la simplicité du modèle à suivre:

Flairer chez les Grecs de «belles âmes», éprises de «juste milieu» et autres perfections, ou, par exemple, admirer chez eux la sérénité dans la grandeur, une âme tournée vers l'idéal, une noble simplicité - voilà une simplicité qui n'est finalement qu'une niaiserie allemande

- écrit Nietzsche dans le Crépuscules de Idoles ${ }^{4}$. Cette niaiserie allemande, elle est l'apanage des philologues allemands - et ici Nietzsche cite un philologue allemand, Christian Lobeck, qui n'a vu que bouffonneries dans les rites dionysiens ${ }^{5}-$ mais il semble bien qu'elle soit également celle de Goethe et de Winckelmann, auxquels il fait allusion plus loin, car eux aussi ont profondément méconnu le dionysiaque et ont ainsi finalement jugé les Grecs à la manière allemande, c'est-à-dire uniquement d'après leurs philosophes ${ }^{6}$. Ils ont en effet mis au premier plan le discours rationnel plutôt que cette expression du trop

\footnotetext{
${ }^{1}$ Lettre du 20 septembre 1803 in: F. Hölderlin, CEuvres, p. 1011.

${ }^{2}$ M. Heidegger, Nietzsche, pp. 119-120.

${ }^{3}$ Cf. F. Nietzsche, Introduction aux études de philologie classique, cours de l'été 1871, p. 102, où Nietzsche refuse l'opposition simpliste d'un pessimisme chrétien à un optimisme païen et déclare: $Q u$ 'on se méfie de l'expression: «sérénité grecque»!

${ }^{4}$ Cf. F. Nietzsche, Crépuscules des Idoles, p. 148.

${ }^{5}$ Cf. F. Nietzsche, Crépuscules des Idoles, p. 150. Christian Lobeck (1781-1860), auteur de l'Aglaophamus, une étude des Mystères dont Nietzsche dira que c'est l'œuvre d'un ver desséché parmi les livres, mais dont Erwin Rohde déclare que c'est un ouvrage toujours digne d'admiration malgré le caractère unilatéral de son rationalisme desséché. Voir à ce sujet Querelle autour de "La naissance de la tragédie”, p. 112 \& p. 191.

${ }^{6}$ Cf. F. Nietzsche, Crépuscules des Idoles, p. 149.
} 
plein de forces ${ }^{1}$ qu'est l'art tragique grec correctement compris, c'est-à-dire perçu comme œuvre du génie dionysio-apollinien et union mystérieuse de ces deux instincts ${ }^{2}$. On ne peut donc comprendre les Grecs, - ce à quoi n'atteint nullement le classicisme selon Nietzsche, qui n'hésite pas à affirmer que Goethe ne comprenait pas les Grecs $^{3}$, - que si on renonce à en faire des modèles et si l'on prend acte de la coupure radicale qui sépare les Anciens des Modernes. C'est ce que Nietzsche fait pour sa part avec la notion de décadence, et ce que font aussi avant lui les postkantiens, Schiller, Hegel et Hölderlin, à l'opposé de Herder et de Goethe, qui ont pu au contraire penser qu'un moderne peut être l'égal d'un ancien et que par exemple Winckelmann n'était rien d'autre qu'un Grec né par hasard au XVIII siècle.

On ne peut en effet aborder la question de cette grécomanie qui s'est emparée de l'Allemagne dans la deuxième moitié du XVIII ${ }^{\mathrm{e}}$ siècle sans évoquer la figure de Johann Joachim Winckelmann, le fondateur de l'archéologie scientifique et de l'histoire de l'art au sens propre de ce terme. Dans ses Réflexions sur l'imitation des ouvres grecques en peinture et en sculpture, publiées à Dresde en 1755, il prône, contre l'art baroque, qui battait alors son plein, le retour au classicisme, c'est-à-dire aux Grecs. Au moment où il arrive à Dresde, qu'il surnommera l'Athènes $d u$ Nord, la Grande Galerie Royale vient d'acquérir un certain nombre de vestiges de l'art grec et romain, et en particulier un moulage en plâtre du groupe du Laocoon, sculpture de la période hellénistique, qui représente l'atroce châtiment infligé par Apollon au prêtre Laocoon et à ses deux enfants, étouffés par de monstrueux serpents. Winckelmann voit dans cette œuvre un sommet de l'art grec et cette même sculpture sera aussi l'objet d'un célèbre essai de Lessing paru en 1766. Ce qui pour Winckelmann caractérise éminemment l'art grec, c'est la noble simplicité, la grandeur sereine et l'expression d'une âme grande et toujours égale ${ }^{4}$. C'est en effet dans la retenue que s'exprime la belle nature grecque, dans laquelle la forme harmonieuse du corps dévoile l'unité et la simplicité de l'âme, et c'est ce que, selon Winckelmann, montre par excellence le Laocoon: Laocoon ne crie pas, il gémit (il soupire) - presque à l'état de repos ${ }^{5}$, car derrière la douleur, il fait apparaitre la force consciente de l'esprit qui cherche à se ressaisir contre elle. Chez les Grecs, selon Winckelmann, la forme révèle l'âme, plastique et éthique ne font qu'un. On comprend à partir de là que Winckelmann ait pu substituer au mot d'ordre traditionnel de l'art, à savoir l'imitation de la nature, un nouvel impératif de la raison, l'imitation des Grecs: L'unique moyen pour nous de devenir grands, voire, si cela est possible, inimitables, est l' imitation des Anciens. ${ }^{6} \mathrm{C}$ 'est en 1764, à Rome, où il séjournera douze ans, qu'il publie son ouvrage le plus

\footnotetext{
${ }^{1}$ Cf. F. Nietzsche, Crépuscules des Idoles, p. 149.

${ }^{2}$ Cf. F. Nietzsche, La naissance de la tragédie § 4, p. 31.

${ }^{3}$ F. Nietzsche, Crépuscules des Idoles, p. 151.

${ }^{4}$ J. J. Winckelmann, Réflexions sur l'imitation ..., pp. 143-147. Voir E. Escoubas, L'Athènes du Nord: Winckelmann à Dresde.

${ }^{5}$ J. J. Winckelmann, Réflexions sur l'imitation ..., pp. 143-147.

${ }^{6}$ J. J. Winckelmann, Réflexions sur l'imitation ..., pp. 96-97.
} 
important, et celui qui aura le plus de retentissement sur le siècle, l'Histoire de l'art de l'Antiquité.

Les idées de Winckelmann trouvent un accueil favorable non seulement parmi ses contemporains, comme Lessing, qui ne lutte contre l'emprise en Allemagne du classicisme français que pour mieux imposer aux Allemands les Grecs comme uniques modèles, mais aussi parmi les poètes et penseurs des générations suivantes: Herder affirme que Winckelmann est un Grec qui a resurgi de la cendre de son peuple pour illuminer notre siècle, Heinse, auteur d'un roman intitulé Ardinghello, paru en 1787, qui aura une grande influence sur Hölderlin, et Goethe lui-même, suivront les traces de Winckelmann et tous deux feront le voyage de Rome, afin de surprendre le secret de l'art des Grecs, ces incomparables artistes. Quant à Friedrich Schlegel, le fondateur en 1798 avec son frère August Wilhelm, de l'Athenaeum, revue qui ne connaîtra que six numéros, mais dans le cadre de laquelle se réuniront ceux que l'on nommera les premiers romantiques - même si, chez celui qui fut l'auteur en 1808 d'un livre célèbre intitulé Sur la philosophie et la sagesse des Hindous, l'indomanie rivalise avec la grécomanie -, il salue pourtant encore dans le saint Winckelmann celui qui fut le premier à avoir trouvé le modèle originel de l'humanité accomplie dans les figures de l'art de l'antiquité ${ }^{1}$.

Comment expliquer la fascination exercée sur tant d'esprits éminents en Allemagne non pas par l'antiquité en général, mais bien par la seule Grèce, alors que les penseurs de la révolution française se tourneront au contraire plutôt vers le modèle de la République romaine? Faut-il à cet égard aller jusqu'à parler de la tyrannie de la Grèce sur l'Allemagne, comme le fait la germaniste anglaise Eliza Butler dans un livre qu'elle écrivit pendant l'accession de Hitler au pouvoir et qui fut publié en $1935^{2}$ ? Dans ce livre qu'elle considérait comme un avertissement, il s'agissait pour elle de retracer le développement du culte rendu à la Grèce ancienne par les écrivains et les penseurs allemands de Winckelmann à Stefan George en passant, entre autres, par Hölderlin ${ }^{3}$, afin de montrer qu'ils étaient demeurés prisonniers d'une image idéalisée de la Grèce, ce retour au paganisme ayant dangereusement contribué à la formation de l'état d'esprit politique des Allemands du XX $\mathrm{X}^{\mathrm{e}}$ siècle. Il y eut par la suite de nombreuses autres études traitant du même sujet, dont en particulier celle de Josef Chytry publiée en 1989 et portant le titre significatif de L'État esthétique ${ }^{4}$, entendant par là le mouvement intellectuel consistant de Winckelmann à Marcuse à tenter de redonner vie à la tradition hellénique de la cité comme site d'une vie à la fois belle et bonne.

Pour comprendre cette hellénophilie allemande, il faut sans doute ici invoquer l'abîme qui sépare la France, nation qui s'est constituée depuis longtemps autour d'un territoire nettement défini, et l'Allemagne qui, depuis la guerre de

Cf. F. Schlegel, Idées, p. 216.

${ }^{2}$ Cf. E. M. Butler, The Tyranny of Grece over Germany.

${ }^{3}$ Dans le chapitre qu'elle consacre à Hölderlin, elle commence par un long récit du voyage en France que Hölderlin fit en 1801-1802 et qui, comme il l'écrivit à son retour à son ami Böhlendorff, lui permit de se familiariser avec la vraie nature des Grecs. Voir à ce sujet A. Warminski, Heidegger en France.

${ }^{4}$ Cf. J. Chytry, The Aesthetic State ... . 
Trente ans et les traités de Westphalie qui la terminent en 1648, est morcelée en une multitude d'États territoriaux et qui va se voir ébranlée, puis anéantie par l'hégémonie française et les victoires napoléoniennes au tout début du XIX ${ }^{\mathrm{e}}$ siècle. C'est dans cette période de crise, au tournant du siècle, que l'idée nationale allemande va se constituer, non pas autour d'un territoire à défendre, mais autour de l'idée de la mission spirituelle qui est dévolue à l'Allemagne, lieu où souffle l'esprit de la poésie et de cette philosophie idéaliste que Fichte, auteur en 1807 des fameux Discours à la nation allemande, a nommée nouvelle. C'est en effet en se tournant vers la Grèce, patrie de l'art et de la pensée, que l'Allemagne peut envisager, dans cette époque d'extrême détresse qui est la sienne, l'espérance d'un monde nouveau. Car ce n'est pas une volonté de régression, de retour en arrière qui anime les premiers romantiques, mais au contraire cette puissante aspiration à un avenir nouveau qu'exprime Novalis, un des participants au cercle de l'Athenaeum, dans l'essai intitulé L'Europe ou la chrétienté, qui date de 1799 , où il en appelle à un total renouvellement du christianisme. C'est Thomas Mann lui-même, peu suspect de complaisance envers l'irrationalisme réactionnaire, qui a dit du romantisme allemand qu'il pouvait être considéré comme le mouvement le plus révolutionnaire et le plus radical de l'esprit allemand ${ }^{1}$. C'est en effet le goût du moderne, de l'actuel et du futur qui constitue à proprement parler le goût romantique, et non la quête d'une origine, dont les romantiques savent bien qu'elle est à jamais perdue. C'est sans doute ce que voulait dire August Schlegel lorsqu'il déclarait dans l'Athenaeum en opposant Winckelmann qui a senti les Grecs comme un Grec à ceux qui savent jeter leur regard aussi bien sur l'ancien que sur le nouveau monde: Peut-être faut-il être archi-moderne pour avoir sur l'antique un point de vue transcendantal. ${ }^{2}$

Comme l'a en effet bien montré Jacques Taminiaux, dans sa thèse publiée en 1967 sur La nostalgie de la Grèce à l'aube de l'idéalisme allemand, dont le sous-titre est: Kant et les Grecs dans l'itinéraire de Schiller, de Hölderlin et de Hegel $^{3}$, il n'y a en effet proprement de nostalgie, c'est-à-dire, selon l'étymologie même de ce terme, forgé au milieu du XVIII siècle, souffrance de l'exil et aspiration au retour, à l'égard de la Grèce que chez ceux qui, dans cette Allemagne du premier romantisme, trouvent encore à l'étranger leurs modèles. Car, comme déjà Kant, ils se nourrissent de l'idée rousseauiste selon laquelle l'état de culture et l'ordre social et politique de ce XVIII ${ }^{\mathrm{e}}$ siècle représentent un écart maximal par rapport à l'état de nature, condition à laquelle l'homme appartient de naissance. Kant propose en effet, dans son opuscule de 1786 Conjectures sur les débuts de l'histoire humaine, une lecture téléologique de l'histoire. Tout se passe, explique-t-il, comme si l'histoire pouvait être comprise comme la mise en œuvre d'un dessein de la nature qui aboutirait, à travers le conflit de la nature et de la liberté, à leur réconciliation finale dans un

\footnotetext{
${ }^{1}$ Th. Mann, Gesammelte Werke in dreizehn Bänden, vol. 10, p. 266.

${ }^{2}$ A. Schlegel, Fragments de l'Athenaeum, p. 138.

${ }^{3} \mathrm{Cf}$. J. Taminiaux, La nostalgie de la Grèce à l'aube de l'idéalisme allemand.
} 
art qui atteignant sa perfection deviendrait de nouveau nature ${ }^{1}$. Comme le souligne Jacques Taminiaux dans un article plus récent: La nostalgie de la Grèce apparaît lorsque Schiller, se plongeant au cours de 1788, dans une lecture fièvreuse et enthousiaste d'Homère et des tragiques grecs, substitue à l'état de nature rousseauiste l'image de la Grèce antique. ${ }^{2}$

On sait en effet que Schiller se plonge en 1788, année de sa rencontre avec Goethe, dans une lecture fiévreuse et enthousiaste d'Homère et des tragiques grecs $^{3}$ qui va être à l'origine de cette nostalgie de la Grèce qui va constituer la tonalité fondamentale de toute la génération suivante. Car pour Schiller, de la Grèce à la modernité, tout s'est profondément transformé, non seulement l'existence humaine mais aussi l'ensemble de la nature, comme l'exprime le poème Die Götter Griechenlandes [Les Dieux de la Grèce], composé en mars de la même année et paru dans Der Teutsche Merkur [Le Mercure allemand], revue fondée par Wieland et qui fut la plus importante d'Allemagne pendant toute cette période ${ }^{4}$. Schiller y dépeignait longuement le temps où les dieux gouvernaient le monde avec les légers liens de la joie, et où l'amour établissait un doux lien entre les hommes, les Dieux et les héros, car comme les dieux étaient encore humains, les hommes étaient plus divins ${ }^{5}$. Ce monde riant a hélas disparu, et aujourd'hui le poète déplore que nulle divinité ne s'offre à [son] regard, car de ces images pleines de vie, hélas, le squelette seul est resté. Dans sa version plus courte parue en 1793, le poème se termine ainsi:

Oisifs, au pays des poètes, les dieux sont retournés

Inutiles à un monde

Qui, ayant dépassé ses lisières,

Se maintient par son propre suspens.

Oui, Ils sont retournés chez eux

Emportant avec eux tout ce qui est grand et beau

Toutes les couleurs, tous les tons de la vie

Et il ne nous est resté que la lettre dépourvue d'âme.

Arrachés au flux du temps

Ils planent, saufs, sur les hauteurs du Pinde;

Ce qui doit vivre éternellement dans le chant,

Va nécessairement dans la vie à sa perte.

Schiller voit en effet dans le christianisme la mort pour ainsi dire charnelle des dieux, du fait que la nature privée de sa divinité, aujourd'hui semblable au battement mort d'un pendule [...] suit servilement la loi de la pesanteur, alors qu'autrefois la plénitude de la vie, tel un flot, traversait toute la création, ce qui fait que l'on ne sentira jamais ce que l'on sentait alors, car tout indiquait le regard béni, tout était la trace d'un dieu. Pour lui, c'est donc l'apparition du

\footnotetext{
${ }^{1}$ Cf. Kant, La philosophie de l'histoire, p. 164.

${ }^{2}$ J. Taminiaux, La nostalgie de la Grèce à l'aube de l'Allemagne classique, p. 33.

${ }^{3}$ J. Taminiaux, La nostalgie de la Grèce à l'aube de l'Allemagne classique, p. 33.

${ }^{4}$ Cf. F. von Schiller, Poèmes Philosophiques.

${ }^{5}$ F. Dastur, Retrait des dieux et modernité selon Novalis et Hölderlin, pp. 31-44.
} 
monothéisme qui met fin à la présence immanente du Dieu dans le monde. L'âge de la médiation commence, mais le médiateur, dans le christianisme, est le corps mort du Christ, c'est cet horrible squelette qui apparaît au chevet du mourant. Dès lors, le Dieu-un n'est plus qu'une image qui se tient, isolée, hors du monde:

Sans amis, sans frère, sans semblable,

Sans déesse, sans fils terrestre,

Un Autre règne dans l'empire de l'éther,

Sur le trône renversé de Saturne.

Bienheureux avant que les êtres s'en soient réjoui,

Bienheureux dans les campagnes dépeuplées,

Il contemple seulement, dans le long flot du temps,

Pour l'éternité - sa propre image.

Schiller n'en reste cependant pas à cette pure déploration, puisque tous les essais philosophiques qu'il va produire dans les années suivantes seront consacrés à l'élaboration d'une problématique visant à surmonter l'épreuve de l'exil et de la nostalgie en permettant à l'homme moderne de trouver la beauté dans la finitude et d'accéder ainsi à nouveau à la lumineuse sérénité des dieux de l'Olympe. Après Grâce et Dignité et Sur le sublime, Schiller publie en 1795 et 1796 dans Les Heures, la revue qu'il a fondée avec Goethe, deux textes dont le jeune Hölderlin prendra aussitôt connaissance, les Lettres sur l'éducation esthétique de l'homme et Sur la poésie nä̈ve et sentimentale. C'est en particulier dans ce dernier texte que l'opposition entre l'hellénité et la modernité est comprise comme la différence entre deux manières de penser et de se rapporter à la nature: est naïf ce qui est nature, est sentimental ce qui cherche une nature perdue. C'est en effet parce que les Grecs vivent en intimité avec la nature que l'on rencontre chez eux peu de traces de l'intérêt sentimental qui attache les modernes aux scènes de la nature. Les Grecs ne font pas de différence entre culture et nature, précisément parce que chez eux la culture se déployait dans la proximité de la nature et sans rupture avec celle-ci. C'est pourquoi Schiller peut dire des Grecs qu'ils sentaient naturellement alors que les modernes sentent le naturel et que le sentiment de la nature chez le moderne ressemble à celui que le malade éprouve pour la santé 1 .

Schiller développe sur cette base une philosophie de l'histoire et voit en celle-ci un progrès qui se réalise dialectiquement en trois moments. Le premier moment est celui, grec, de l'harmonie entre la nature et l'art, un art qui, comme le dit Aristote dans sa Physique, imite ou parachève la nature. Dans le second moment, moderne, l'art conquiert sa liberté en se retournant contre la nature et en brisant les liens qui l'unissent à la totalité. On a donc affaire à une antithèse entre le premier et le second moment, entre la poésie naïve et la poésie sentimentale, entre les poètes anciens et les poètes modernes, entre la réalité et l'idéal, puisque si les poètes anciens sont nature, les poètes modernes en cherchant la nature perdue font de celle-ci un idéal. Le troisième moment est celui où, conformément à ce que prescrivait Kant comme fin dernière de la

${ }^{1}$ F. von Schiller, Poésie naïve et poésie sentimentale, pp. 101-103. 
destination morale pour l'espèce humaine ${ }^{1}$, l'art achevé retourne à la nature et où se concilient la sensibilité et la raison, la nature et la liberté. Il y a donc une logique de l'histoire: la nature doit être anéantie par son opposé, l'art destructeur, pour se retrouver dans la synthèse idéale de l'art et de la nature qui advient comme accomplissement total de l'essence de l'homme qui est un être à la fois réceptif et spontané, naturel et libre, comme Kant l'a bien montré. Car ce que cherche Schiller, qui ouvre avec Fichte l'ère du post-kantisme, c'est une synthèse réconciliatrice qui permette le dépassement du dualisme kantien de la nature et de la liberté et aboutisse ainsi à un dépassement de la nostalgie et à la possibilité de trouver la beauté dans la finitude afin de faire accéder les mortels à la lumineuse sérénité des dieux de l'Olympe.

Il n'est pas douteux que Hegel et Hölderlin sont partis eux aussi des mêmes références que Schiller, comme l'atteste en particulier une lettre de Hölderlin qu'il écrit du séminaire de Tübingen en juillet 1793 à son ami Neuffer et comme il le répète un an plus tard dans une lettre à Hegel datée du 10 juillet 1794: Kant et les Grecs sont à peu près ma seule lecture. On peut dire en effet que les deux amis ont, dès le début de leur séjour à Tübingen, vu tous deux dans la Grèce antique à la fois une image idéale, une utopie, et ce qu'il faut opposer comme contrepoint à la détresse allemande actuelle. Hölderlin est en cela le maître de Hegel, il l'introduit aux Grecs, lui qui sait le grec bien mieux que Hegel, et s'intéresse aux tragiques, Euripide et surtout Sophocle. L'amitié de Hölderlin et de Hegel s'est en effet développée à Tübingen sous la forme d'un commun intérêt pour les tragédies de Sophocle. Hegel se propose de traduire Antigone, car il voit dans cette tragédie le caractère politique de la religion grecque, la religion constituant ici la réalisation de la morale pour l'ensemble de la société, alors que le christianisme n'est qu'une religion privée. A cette époque Hölderlin voit dans la Grèce l'esprit de la beauté, demeurant encore très proche en cela de Winckelmann et de Schiller. Pour Hegel aussi, la Grèce représente l'âge d'or, car elle est la nation esthétique, alors que le christianisme engendre le despotisme ou du moins en est complice, car il dénature l'homme en voulant l'élever jusqu'aux cieux. La Grèce, c'est donc la nature, la beauté, la liberté et la joie, celle des fêtes populaires et religieuses (Hegel pense aussi sans doute aux fêtes révolutionnaires), dans lesquels les intérêts privés et publiques s'unissent, alors que les rites du christianisme (c'est-à-dire du protestantisme) ont la couleur du deuil.

Lors des années passées au séminaire, Hegel a développé l'idée d'une religion populaire, similaire à celle des Grecs qui était une religion de la beauté et de l'imagination. Mais après son départ du séminaire, son enthousiasme pour les anciens Grecs se tempéra. À Berne il commença à se tourner vers la philosophie pratique de Kant. Ce qui a éveillé son intérêt, c'est surtout l'un des derniers livres de Kant, La religion dans les limites de la simple raison, paru en 1793, dans lequel Kant oppose au christianisme en tant que religion révélée une religion rationnelle fondée sur l'autonomie et la moralité de l'être humain. C'est de là que part sa critique du christianisme, qui se développe selon deux

${ }^{1}$ I. Kant, Conjectures sur les débuts de l'histoire humaine, p. 164. 
directions, par comparaison avec la religion grecque d'une part, et par comparaison avec le rationalisme moral de Kant d'autre part. Cette critique du christianisme est un appel à lui substituer une religion pratique, morale, qui est la vraie religion de Jésus par opposition au christianisme officiel, mais aussi au judaïsme qui reste enfermé dans le formalisme du rite. Mais on y trouve également une critique du christianisme au nom d'une religion populaire dont le modèle est la religion grecque en tant que religion d'un peuple formant une totalité vivante. Le projet de Hegel est en effet à cette époque celui de la conciliation de la religion morale et de la religion populaire tel qu'on le trouve dans le fameux Systemprogramm ${ }^{1}$. Où faut-il donc chercher une religion qui soit populaire? Du côté de cette simplicité des mœurs qui se perd avec les progrès de la raison ${ }^{2}$, une religion populaire étant celle qui exprime la foi du peuple en sa propre force, qui parle à l'imagination et exalte la vie. C'est en effet ce qui préserve le peuple de la grande inégalité des classes, car l'histoire s'est développée sur le propre sol du peuple, et les légendes qui la font connaître, précisément parce qu'elles ne sont pas issues d'un pays étranger sont en une égale mesure la propriété de chacun ${ }^{3}$. Hegel penche plutôt, on le voit, du côté du cœur et du peuple. Mais il ne pense pas que religion morale et religion populaire sont absolument incompatibles, et ce qui l'atteste pour lui, c'est la conciliation de la raison et du cœur qu' incarne le personnage de Socrate qu'il oppose à celui du Christ, opposition qu'il reprend en 1796 dans La positivité de la religion chrétienne ${ }^{4}$.

Dominique Janicaud, dans l'ouvrage remarquable qu'il a publié en 1975 sous le titre Hegel et le destin de la Grèce a montré non seulement que c'est à partir de l'idéal grec que s'organisent les premières pensées de Hegel, lecteur de Winckelmann et de Lessing, mais aussi comment le thème grec, si présent à l'époque de Francfort, où il partage encore une véritable proximité philosophique avec Hölderlin, ne cesse de nourrir toute l'œuvre ultérieure du penseur, dont il faut rappeler qu'il ouvre encore en 1823 ses leçons de Berlin sur 1'histoire de la philosophie grecque par ces mots: Grèce: à ce nom, le cœur de l'homme cultivé d'Europe, et de nous Allemands en particulier, se sent en terre natale. Dans son ouvrage Dominique Janicaud fait clairement apparaître que, si Hegel rompt avec l'hellénisme littéraire de son époque, en laissant de côté la notion d'imitation, il n'en demeure pas moins que son image de la Grèce demeure celle, idéalisée, de Winckelmann, de Goethe et de Schiller, c'est-àdire la patrie de la beauté sans doute, mais d'une beauté harmonieuse, ordonnée, à dominante apollinienne ${ }^{5}$.

${ }^{1}$ Rappelons que ce texte de deux pages est un fragment autographique de la main de Hegel écrit au tout début de 1797, mais qui a servi de base à une discussion entre Schelling, Hölderlin et Hegel. Sa publication en 1917 par Franz Rosenzweig a donné lieu à tout un débat philologique concernant l'identité de son véritable auteur. Il faut souligner que c'est lui qui lui donna ce titre, lequel semble suggérer qu'il s'agit là de la matrice de ce qui deviendra, essentiellement avec Hegel, l'idéalisme allemand.

${ }^{2}$ G. W. F. Hegel, Fragments de la période de Berne, p. 44.

${ }^{3}$ G. W. F. Hegel, La positivité de la religion chrétienne, pp. 43-44.

${ }^{4}$ Cf. F. Dastur, Leçons sur la genèse de la pensée dialectique, pp. 17-29 \& pp. 155-171.

${ }^{5}$ D. Janicaud, Hegel et le destin de la Grèce, p. 329. 
C'est donc plutôt vers Hölderlin que je me tournerai maintenant pour tenter de montrer que c'est seulement avec lui, mais à travers un long itinéraire, qu'à cette image idéalisée d'une Grèce apollinienne va se substituer l'image complexe d'une Grèce profondément divisée en elle-même. À l'époque où il déclare à Hegel que les Grecs et Kant constituent ses seules lectures, Hölderlin travaille à une première ébauche de son roman qui sera publié en novembre 1794 dans Thalia, la revue de Schiller et qui s'ouvre précisément sur une reprise de l'idée kantienne de l'opposition de la nature et de la culture:

Il y a pour l'homme deux états idéaux: l'extrême simplicité où, par le seul fait de l'organisation naturelle, sans qui nous y soyons pour rien, nos besoins se trouvent en accord avec eux-mêmes, avec nos forces et l'ensemble de nos relations; et l'extrême culture, où le même résultat est atteint, les besoins et les forces étant infiniment plus grands et plus complexes, grâce à l'organisation que nous sommes en mesure de nous donner. ${ }^{1}$

Hölderlin, qui parle un peu après de l'orbite excentrique qui mène de la nature à la culture, affirme donc qu'il y a une identité entre l'état originaire de l'humanité et l'état de son accomplissement le plus achevé, au sens où l'homme doit apprendre à retrouver par l'organisation qu'il se donne à lui-même cet accord avec soi-même qui était le fait de la nature. Mais cette exigence d'une union supérieure, d'une hyperculture qui ferait retour à la nature, comme le prescrit Kant, est difficile à réaliser. Ce qui anime en effet Hypérion, c'est l'esprit d'impatience et ce qu'il lui faut donc apprendre, c'est que le retour à cette belle totalité que Hölderlin nomme à la fois la nature et le sacré, et à laquelle la Grèce antique a su correspondre si harmonieusement ne peut être aujourd'hui immédiat. Car ce qui guide encore ici Hölderlin, c'est la formule qu'il a découvert en lisant le livre que Jacobi a consacré à Spinoza: Hen kai Pan [l'Un et le Tout], qui deviendra le mot de passe réunissant les trois amis du séminaire de Tübingen, Schelling, Hegel et Hölderlin, et plus tard aussi le titre d'un poème de Goethe (Eins und Alles).

À cette époque, le spinozisme se confond pour lui avec le sentiment panthéiste d'un dieu identifié à la totalité de la nature dont Hölderlin trouve l'exemple dans le Phèdre et le Timée de Platon. C'est en effet en 1793, au moment où il rédige la première ébauche de son roman (Fragment Thalia), que Hölderlin, écrivant à son ami Neuffer, évoque ces heures divines où (il) revient du sein de la glorieuse nature ou du bois de platanes au bord de l'Ilisos et où allongé parmi les disciples de Platon [...] (il) l'a suivi au plus profond des profondeurs, aux sources les plus lointaines de l'esprit, où l'âme du monde projette sa vie dans les milles pulsations de la nature, d'où les forces s'écoulent et où elles reviennent après leur immense révolution ${ }^{2}$. Mais cette aspiration à la totalité qui fait dire à Hypérion, toujours dans le fragment Thalia: Ce qui ne

\footnotetext{
${ }^{1}$ In: F. Hölderlin, Euvres, p. 113.

${ }^{2}$ In: F. Hölderlin, Euvres, p. 91.
} 
peut m'être tout, pour l'éternité, ne m'est rien se heurte à la réalité de la finitude et de la séparation qu'elle ne peut abolir durablement. Certes l'apparition de l'héroïne du roman, Mélite, qui incarne la belle totalité, qui est donc le sacré même devenu visible, est cet événement inexprimable qui est celui d'une délivrance de la finitude: Mon existence terrestre était morte, le temps n'était plus; délivré de ses chaînes, proprement ressuscité, mon esprit pressentait sa race et son origine. ${ }^{1}$

Mais cette apparition immédiate du sacré, d'une totalité qui demeure extérieure au fini et est donc une fausse totalité et un faux infini, n'est pas appropriable par l'être séparé qui s'efforce de s'unir à elle. C'est pourquoi à la révélation du sacré succède inexorablement la conscience douloureuse de la séparation qui se trouve comme exaspérée par cette révélation. La première ébauche du roman n'en dit pas plus et s'achève sur le départ de l'aimée auquel succède une profonde détresse pour Hypérion qui ne cherche plus qu' à (se) retrancher du monde des vivants', mais qui pourtant perçoit l'appel que lui adresse le monde: pourquoi me dédaignes-tu? ${ }^{2}$, ce qui laisse entendre que commence à se révéler à lui la nécessité d'endurer la séparation, et de ne pas en rester à l'antithèse non résolue de l'Un-tout et de la finitude, de la patrie et de l'exil, de la Grèce et de la modernité.

Dans la version définitive du roman, si le but d'Hypérion demeure bien l'union avec la nature, en un tout infini, comme le dit la préface, ce but n'est plus visé dans l'impatience qui exige l'immédiateté du sacré, car l'unique totalité que recherche Hypérion n'est plus l'au-delà d'une identité idéale mais, comme le souligne bien Jacques Taminiaux ${ }^{3}$, la présence actuelle d'une beauté qui se déploie dans le sensible. Voici en effet comment Hypérion décrit l'apparition de l'héroïne qui a maintenant pour nom Diotima:

Je fus heureux une fois, Bellarmin! Ne le suis-je pas encore? Ne le serais-je pas, même si le moment sacré où je la vis pour la première fois avait été le dernier? Je l'aurai vue une fois, l'unique chose que cherchait mon âme, et la perfection que nous situons au-delà des astres, que nous repoussons à la fin des temps, je l'ai sentie présente. Le bien suprême était là, dans le cercle des choses et de la nature humaine.

Je ne demande plus où il est: il fut dans le monde, il y peut revenir, il n'y est maintenant qu'un peu plus caché: je l'ai vu et je l'ai connu.

$\hat{O}$ vous qui recherchez le meilleur et le plus haut, dans la profondeur du savoir, dans le tumulte de l'action, dans l'obscurité du passé ou le labyrinthe de l'avenir, dans les tombeaux ou au-dessus des astres,

\footnotetext{
${ }^{1}$ In: F. Hölderlin, CEuvres, p. 116.

${ }^{2}$ In: F. Hölderlin, Euvres, p. 131 \& p. 132.

${ }^{3}$ L'analyse ici proposée du roman s'appuie sur la lecture qu'en fait J. Taminiaux, La nostalgie de la Grèce à l'aube de l'idéalisme allemand, pp. $131 \mathrm{sq}$. Voir également F. Dastur, Roman et philosophie: l'Hypérion de Hölderlin, pp. 293-306.
} 
savez-vous son nom? le nom de ce qui constitue l'un

et le tout?

Son nom est beaute. ${ }^{1}$

La beauté, ce n'est donc pas cette unité qui exclut la différence et qui se tient au-delà du fini, mais au contraire une totalité qui inclut le fini et s'annonce à partir de lui. Cette beauté, plus encore que platonicienne, est héraclitéenne, car elle se donne dans le devenir et non pas dans l'éternité de l'idée. C'est pourquoi Hypérion, faisant l'éloge de la Grèce et de la beauté, est amené à évoquer un passage du Banquet de Platon où est cité le fragment 178 d'Héraclite qui parle de l'accord de ce qui diffère d'avec soi et de la palintropos harmoniê, de l'harmonie de sens opposé de l'arc ou de la lyre:

Seul un Grec pouvait inventer la grande parole

d'Héraclite, hen diapheron eautô - l'un distinct en soi-

même -, car elle dit l'essence de la beauté, et avant

qu'elle fût inventée, il n'y avait pas de philosophie. ${ }^{2}$

$\mathrm{C}^{\prime}$ est ici que Hölderlin conquiert un rapport non nostalgique à la Grèce, qui n'est plus d'aspiration à une impossible plénitude, comme il l'écrit à son ami Neuffer en 1794, affirmant qu'il commence à surmonter cette lâcheté puérile qui le pousse à se plaindre éternellement que le monde ne soit pas une Arcadie ${ }^{3}$. Car ce qui est ainsi surmonté, comme le souligne justement Jacques Taminiaux, c'est l'antithèse schillérienne de la lumière grecque et des ténèbres modernes et ce surmontement engage un rapport aux Grecs qui ne sera plus placé sous le signe de la nostalgie de l'Hen kai Pan, mais de la méditation grave de l'Hen diapheron eautô ${ }^{4}$.

La philosophie n'est donc pas née de la nostalgie d'une unité absente ni de l'exil hors du tout, mais d'un accord avec ce qui est dans la différence de sa multiplicité. Car ce qui est ainsi atteint, c'est un autre concept de la beauté que celui du platonisme et du classicisme de Goethe et de Winckelmann: non plus le devenir visible de l'idée, mais l'harmonie des contraires, le concept non plus statique d'une beauté intemporelle, mais celui, dynamique, d'une beauté vivante que Platon lui-même, qui cite Héraclite, n'a d'ailleurs peut-être pas ignoré, comme Hölderlin le laisse entendre dans la Préface d'Hypérion, lorsqu'il s'exclame, après avoir fait allusion à la présence déjà réalisée de l'être comme beauté:

Je crois qu'à la fin nous nous écrierons tous: saint

Platon, pardonne-nous! nous avons gravement péché contre toi!.$^{5}$

Car c'est à partir d'une telle présence sensible de la beauté et de l'existence effective de l'union du fini et de l'infini qu'est définie dans Hypérion la Grèce en tant que patrie de la philosophie par opposition à l'Égypte et au Nord:

\footnotetext{
${ }^{1}$ In: F. Hölderlin, Euvres, p. 177.

${ }^{2}$ In: F. Hölderlin, Cuvres, p. 203.

${ }^{3}$ In: F. Hölderlin, Euvres, p. 323.

${ }^{4}$ J. Taminiaux, La nostalgie de la Grèce à l'aube de l'Allemagne classique, p. 43.

${ }^{5}$ In: F. Hölderlin, CEuvres, p. 1150.
} 
Comprenez-vous maintenant pourquoi les Athéniens, plus que quiconque, devaient être aussi un peuple philosophe?

L'Égyptien ne le pouvait pas. Qui n'aime pas le ciel et la terre et n'est pas aimé d'eux, qui ignore l'accord avec l'élément dans lequel il vit, accord dispensé par ce mutuel amour, ne peut être, par nature, en accord avec soi; et s'il ressent la beauté éternelle, ce ne peut être, en tout cas, avec l'aisance des Grecs. ${ }^{1}$

Seule en effet la Grèce est capable de cet accord avec le sensible et l'extériorité, qui lui procure l'accord avec l'intelligible et l'intériorité: ni l'Oriental (l'Égyptien), assujetti à une extériorité qui apparaît comme une terrible énigme, ni le Nordique (l'Allemand), enfermé dans une intériorité sans dehors, ne sont capables d'un tel accord et ne peuvent s'ouvrir à une beauté à la fois humaine et divine. Faut-il donc ressusciter la Grèce? L'échec de l'expédition guerrière en vue de la libération de la Grèce à laquelle participe Hypérion aux côtés de son ami Alabanda atteste au contraire que c'est là seulement un rêve. Car cet accord avec le tout ne peut être arraché par violence, ni représenté dans la nostalgie. Il ne peut qu'être réalisé par l'acceptation et l'endurance de la séparation. Tel est le sens des paroles d'Hypérion dans une des dernières lettres du roman:

Cher ami, je suis calme, car je n'exige pas un sort meilleur que les dieux. Toutes choses ne doivent-elles pas souffrir? Et d'autant plus qu'elles sont plus excellentes? La sainte Nature ne souffre-t-elle pas? $\hat{O}$ ma divinité! Que tu puisses souffrir, radieuse comme tu l'es, j'ai mis longtemps à l'admettre. Mais la félicité qui ignore la souffrance est sommeil, et sans mort, il n'est pas de vie. ${ }^{2}$

L'accent est donc mis sur la différence, sur la souffrance de la séparation: ni l'humanité ni la nature ne coïncident avec elles-mêmes. Et la Grèce ellemême, dont est donnée dans le roman une vision dynamique, ne peut pas davantage exclure de soi la division. C'est pourquoi la Grèce va devoir moins s'opposer de l'extérieur à cette figure de la séparation qu'est l'Orient égyptien, dont Hölderlin trouve le modèle chez Winckelmann et qui annonce la figure du juif des écrits francfortois de Hegel, qu'intégrer profondément en soi-même une autre figure de l'Orient, extatique et dionysiaque, que Hölderlin nomme aussi aorgique, mot qu'il emprunte à Schelling. Il semble en effet que l'Orient s'annonce sous cette double figure (figure de la séparation et figure de l'illimitation) à chaque fois qu'un excès dans un sens ou dans un autre - trop de culture et d'art et donc trop de séparation avec la nature ou trop d'enthousiasme et donc trop d'union avec le tout ${ }^{3}-$ menace. Ainsi à la figure d'Empédocle qui incarne

\footnotetext{
${ }^{1}$ In: F. Hölderlin, Euvres, p. 203.

${ }^{2}$ In: F. Hölderlin, Euvres, p. 264.

${ }^{3}$ Il faudrait rappeler ici le thème de l'hyperbole, c'est-à-dire de l'unification par opposition extrême, qui commande toute l'exposition de l'individualité poétique dans le grand essai de Hölderlin intitulé De la démarche
} 
l'esprit d'impatience et le désir spéculatif d'une union prématuré avec la totalité s'oppose celle de l'adversaire, celle du prêtre égyptien Manès, celui qui demeure, qui met en question le droit d'Empédocle à concilier les oppositions et qui personnifie la capacité d'endurer le maintien de la tension entre art et nature. Mais à cette dernière figure, comme à celle d'Edipe qui se voit renvoyé au monde terrestre et à une mort seulement spirituelle, une mort sans mort, s'oppose celle d'Antigone qui accomplit le retournement natal du grec qui la ramène de la sobriété européenne à l'élément oriental du feu céleste. Car le contexte dans lequel se situe Hölderlin est celui de la Querelle des Anciens et des Modernes qui éclata en France à la fin du XVII ${ }^{\mathrm{e}}$ siècle et que Goethe et Schiller ont réactivée en Allemagne à la fin du XVIII ${ }^{\mathrm{e}}$ siècle pendant la période de ce mouvement littéraire qu'on a nommé le Sturm und Drang [Tempête et passion]. Le problème essentiel qui se posait alors est, comme nous l'avons déjà vu, celui de l'imitation des Grecs qui sont considérés comme le sommet de l'accomplissement artistique. L'opposition des Anciens et des Modernes avait déjà constitué le thème du roman Hypérion et la conclusion d'Hölderlin était alors que cette opposition demeurait insurmontable, à cause de l'infranchissable distance qui nous sépare des Anciens. C'est ainsi que dans la première lettre de Hölderlin à son ami Casimir Böhlendorf, il est dit qu'il ne nous est pas du tout permis d'avoir avec eux (les Anciens) quelque chose d'identique'. Dans un court essai de 1799 intitulé De quel point de vue faut-il considérer l'Antiquité? Hölderlin insiste sur la différence entre les Grecs et les Modernes, en soulignant le caractère trop écrasant de l'héritage qu'ils nous ont légué. Nous autres modernes sommes entièrement subjugués par l'exemplarité de l'Antiquité. Hölderlin parle à ce propos de notre sujétion à l'égard de l'Antiquité, laquelle apparaît comme quelque chose de positif, qui a déjà été approprié, et suggère que nous n'avons guère à choisir qu'entre être écrasés sous le point de l'acquis ou nous opposer violemment à lui. Mais en fait combattre contre ce qui a déjà été approprié signifierait se lier à lui de manière encore plus étroite. Car ce qui fait véritablement problème dans notre rapport à l'antiquité, c'est qu'elle ne peut pas servir de matériel éducatif pour nous, précisément parce qu'elle est déjà formée et que notre tendance formatrice nous incite à rechercher un matériau brut que nous pourrons former nous-mêmes:

Le pire, c'est que l'Antiquité semble tout à fait opposée

à notre instinct spontané qui tend à former l'informe,

à perfectionner le spontané, le naturel, et celui qui est né pour l'art préfère naturellement ce qui est brut, inculte, enfantin, plutôt qu'une matière déjà formée qui anticipe sur ce qu'il entend former lui-même. ${ }^{1}$

Hölderlin dit ici explicitement que l'Antiquité apparaît comme opposée aux Modernes que nous sommes et que c'est cette opposition même qui est le plus

de l'esprit poétique. Il s'agit pour l'individualité de passer d'un trop d'objectivité ou d'un trop de subjectivité à l'opposition harmonique du moment objectif et du moment subjectif dans l'état accompli de sa formation qui est celui de la création poétique.

${ }^{1}$ In: F. Hölderlin, Euvres, p. 594. La traduction citée ici est celle de F. Fédier in: F. Hölderlin, Remarques sur Edipe, Remarques sur Antigone, pp. 97-99. 
difficile (das Schwerste) dans notre relation à elle. Il est clair que Hölderlin ne partage pas cette opinion commune qui voit dans l'Antiquité l'enfance ou la jeunesse de l'humanité, mais qu'il considère au contraire que l'Antiquité est excessivement formée. Il remarque d'ailleurs que la raison générale du déclin des peuples vient précisément de cet excès de formation qui pétrifie leur vivante originalité en des formes positives et conduit leur authenticité à se perdre dans le luxe. Ce fut là en effet la cause du déclin de la Grèce comme le dit un fragment d'un hymne tardif:

$$
\begin{aligned}
& \text { [...] nommément ils voulaient instaurer } \\
& \text { Un règne de l'art. Par là pourtant } \\
& \text { Le natal par eux } \\
& \text { Fut chômé et pitoyablement alla } \\
& \text { Le pays grec, le plus beau, à sa perte. }
\end{aligned}
$$

Ainsi les Grecs n'ont-ils pas été capables de faire retour à ce qui leur était proprement natif (vaterländisch), ils ont péri d'un excès d'art parce qu'ils ne parvinrent pas à concilier en eux-mêmes la nature et la culture, ce qui aurait signifié le renouvellement incessant de l'une par l'autre. Cependant ce qui caractérise les modernes comme les Grecs, c'est cette tendance constante de la nature humaine que Hölderlin, en reprenant un concept alors à la mode, nomme tendance formatrice (Bildungstrieb). Celle-ci a pourtant, replacée dans le contexte historique, une direction différente dans l'antiquité et dans la modernité. C'est précisément ce que Hölderlin explique dans sa première lettre à Böhlendorff:

Nous n'apprenons rien plus difficilement que le libre usage du nationel. Et je le crois, c'est justement la clarté de la représentation qui nous est originellement aussi naturelle qu'aux Grecs le feu du ciel. C'est précisément pourquoi ils seront surpassables plutôt dans l'éclat de la passion - que tu as également su te conserver - que dans leur homérique présence d'esprit qui est don de présentation.

Cela sonne comme un paradoxe. Mais je l'affirme encore une fois et je le livre à ton examen pour que tu en fasses usage; avec le progrès de la culture, ce qui est proprement nationel perdra toujours plus de sa primauté. C'est pourquoi les Grecs sont moins maîtres du pathos sacré, parce qu'il leur était inné; ils excellent au contraire dans le don de présentation, à partir d'Homère, parce que cet homme extraordinaire avait assez de force d'âme pour ramener à son empire d'Apollon la proie de la sobriété junonienne et occidentale, s'appropriant ainsi vraiment l'élément étranger.

Chez nous c'est l'inverse. ${ }^{1}$

\footnotetext{
${ }^{1}$ In: F. Hölderlin, Euvres, p. 1003.
} 
De ces lignes nous pouvons conclure qu'il y a une inversion dans la relation de la nature et de la culture entre les Grecs et nous. Ce qui est leur nature (orientale), le pathos sacré et le feu du ciel, est pour nous culture. Et ce qui est leur culture (occidentale), la clarté de la présentation et la sobriété junonienne, est pour nous nature. Chez tous les peuples la tendance formatrice mène à l'élément étranger, de sorte que ce qui est propre et naturel tend à être oublié et laissé non maîtrisé. C'est pourquoi les Grecs peuvent être surpassés dans l'éclat de la passion qui est leur nature, mais non pas en ce en quoi ils excellent, dans leur don de présentation.

Homère est le Grec par excellence parce qu'il a été capable de s'approprier complètement l'élément étranger, c'est-à-dire le principe occidental de limitation, de différenciation, principe auquel Hölderlin attache aussi le nom de Junon, pour marquer son caractère éminemment terrestre. Quand donc l'art grec fait montre de mesure et de clarté, cela ne nous enseigne rien sur la nature grecque, au contraire de ce qu'avaient pu croire Goethe et Winckelmann, car cette nature est au contraire orientale ou aorgique. L'aorgique ou anorganique, c'est le principe de la nature dans son unicité infinie en tant qu'elle est sans forme et sans organisation, alors que l'organique, c'est le principe de l'art qui suppose une organisation interne, une opposition des parties.

Dans sa seconde lettre à Böhlendorff datée du 2 décembre 1802, Hölderlin définit le caractère du peuple grec comme l'art d'accueillir des natures étrangères et se communiquer à elles ${ }^{1}$. Ce qui caractérise donc les Grecs, c'est la tendresse, l'ouverture à l'étrangeté, tandis que ce qui caractérise les modernes, c'est la sobriété propre à l'individualité close sur elle-même. On comprend dès lors que les Grecs aient à se protéger eux-mêmes contre leur tendresse illimitée à l'aide du principe étranger de différenciation. C'est pourquoi dans la même lettre, écrite de Bordeaux, où il est alors précepteur du consul d'Allemagne, Hölderlin déclare que la nature athlétique des gens du sud, dans les ruines de l'esprit antique, [lui] a rendu l'être propre des Grecs plus familier. Ce qui est proprement grec, c'est la nécessité d'appliquer le principe athlétique de la forme afin de se protéger soi-même de l'excès du feu oriental qui constitue sa nature, alors que chez les modernes, c'est précisément l'inverse.

Par là s'éclaire ce que Hölderlin disait dans sa première lettre à Böhlendorff:

Voilà pourquoi il est également si dangereux de tirer les règles de notre art de la seule perfection grecque. $J$ 'y ai longtemps peiné et je sais désormais qu'à part ce qui doit être, chez les Grecs et chez nous, le plus haut, à savoir le rapport vivant, le destin, il ne nous est pas du tout permis d'avoir avec eux quelque chose d'identique.

Mais ce qui est propre doit tout aussi bien être appris que ce qui est étranger. C'est pourquoi les Grecs nous sont indispensables. Seulement nous ne

\footnotetext{
${ }^{1}$ In: F. Hölderlin, Euvres, p. 1010.
} 
pourrons pas les rejoindre précisément dans ce qui nous est propre, nationel, parce que, encore une fois, le libre usage de ce qui nous est propre est ce qu'il y a de plus difficile. ${ }^{1}$

Nous ne pouvons pas simplement imiter les Grecs parce que notre art doit répondre à une nature qui est diamétralement opposée à la leur. Nous devons nous approprier ce qui leur est naturel, le pathos sacré et le feu du ciel, exactement comme ils devaient s'approprier ce qui nous est naturel, la clarté de la présentation et la sobriété junonienne. On voit par là que Hölderlin s'oppose au classicisme pour lequel l'art grec est la norme parfaite de tout art à venir. Selon lui, nous devons être modernes et ne pas chercher nos modèles dans l'antiquité. Mais nous avons pourtant quelque chose en commun avec les Grecs, qui n'est ni la nature, ni la culture, mais est plus haut que l'une et l'autre qui n'en sont que des éléments abstraits: das lebendige Verhältnis und Geschick, le rapport vivant et le destin ou l'adresse, lesquels impliquent que nous ayons comme eux à nous approprier ce qui nous est étranger. C'est pourquoi, en dépit du fait que les Grecs ne puissent ni ne doivent être imités, ils nous demeurent cependant indispensables. Car nous avons encore à apprendre l'usage de ce qui nous est propre, c'est-à-dire la clarté de la présentation et la sobriété junonienne que les Grecs ont maîtrisé en tant que c'était là pour eux l'élément étranger de leur culture. De sorte que les Grecs ne peuvent pas nous assister dans notre art, mais parce que l'art grec nous donne une image de notre nature, il peut nous aider à accomplir ce que les Grecs ont manqué pour eux-mêmes, l'atteinte au libre usage de ce qui est propre. Les Grecs peuvent ainsi nous aider à accomplir ce que Hölderlin, dans les Remarques sur Sophocle, nommera die vaterländische Umkehr, le retournement natal.

L'originalité de la conception hölderlinienne du rapport de l'antiquité à la modernité vient de ce que pour Hölderlin le Grec ne diffère pas simplement de l'Hespérien comme la nature (l'enfance) de la culture (la maturité), mais que chacun d'eux est divisé en lui-même entre nature et culture, phusis et tekhnè. Hölderlin n'oppose pas, comme Schiller le fait encore, les Grecs aux Modernes de manière externe. C'est pourquoi il ne s'agit pas pour lui de choisir entre le parti des Grecs et celui des Modernes, entre le passé et l'avenir. Les Grecs sont en quelque sorte un miroir inversé de nous-mêmes, ils ne représentent pas quelque chose d'un passé révolu. Car ils ont plus ouvert des possibilités de vie que produit des œuvres qui devraient être imitées. C'est pourquoi ils demeurent un exemple alors même qu'il apparait clairement qu'ils ne peuvent ni ne doivent être imités. Il faut en effet distinguer le modèle de l'exemple, ce qui a à être imité en un sens statique et reproductif de ce qui peut être suivi de manière dynamique et inventive ${ }^{2}$. Nous pouvons tirer une leçon de l'échec des Grecs en ce sens que ce qui a causé leur ruine, l'obsession de la forme, peut avoir pour

\footnotetext{
p. 99.

${ }^{2}$ Voir à ce propos B. Alleman, Hölderlin entre les Anciens et les Modernes, p. 315, qui cite l'épigramme de Klopstock intitulé Solution du doute: Imiter m'est interdit, et pourtant me nomme / Ta sonore louange toujours et encore la Grèce. / Si le Génie en ton âme est ardent / Alors imite le Grec. Le Grec inventait.
}

${ }^{1}$ In: F. Hölderlin, Euvres, pp. 1003-1004. F. Hölderlin, Remarques sur Edipe, Remarques sur Antigone, 
nous la valeur d'un exemple négatif qui peut nous inciter à retourner notre tendance culturelle vers l'illimité dans la direction opposée et la diriger vers notre nature terrestre. Nous ne devons pas imiter leur art et leur culture, mais nous pouvons néanmoins suivre leur exemple de sorte que nous fassions retour à notre propre nature et accédions à cette hyperculture qu'est l'apprentissage du libre usage de ce qui nous est propre. C'est donc dans leur échec même que les Grecs demeurent un exemple pour nous modernes.

C'est à partir de là qu'on peut tenter de comprendre le projet du dernier Hölderlin, celui de la traduction des tragédies de Sophocle. Car il s'agit clairement dans cette entreprise de tenter de mieux se comprendre soi-même et son époque en faisant ce détour par l'étranger que Hölderlin a aussi réellement entrepris par son voyage à Bordeaux. Car, comme le note Wolfgang Binder dans son article sur Hölderlin et Sophocle ${ }^{1}$, il existe une troisième voie entre l'académisme mimétique et la rupture avec la tradition, c'est celle qui conduit Hölderlin, comme il l'écrit à Schiller en 1801, à chercher à se libérer de l'asservissement à la lettre du grec en montrant que la grande précision des auteurs grecs vient de leur plénitude d'esprit ${ }^{2}$, en d'autres termes en faisant apparaître que l'esprit aorgique et porté vers l'illimité des Grecs a dû se donner dans son art la forme la plus stricte pour pouvoir se saisir soi-même, ce qui est le problème même des Grecs, comme le dit Hölderlin dans les Remarques sur Antigone, alors que celui des Modernes est au contraire, dans l'absence de partage qui les caractérise, et de par leur naissance dans un monde de convention, où tout est rigidement structuré en disciplines et en cases, de pouvoir atteindre quelque chose. Il s'agit donc à la fois de faire apparaitre l'originelle nature orientale des Grecs que ceux-ci ont chômé en se perdant dans ce que Schiller nomme le lointain pays de l'art et de faire sortir ces Modernes que Hölderlin nomme plus précisément des Hespériens, des habitants du couchant, des Occidentaux, de leur monde natal de convention en leur ouvrant la possibilité d'accomplir leur destin et de s'ouvrir ainsi à l'étrangeté orientale de leur culture. Car la reconduction du grec à son propre coïncide avec l'accomplissement culturel hespérique, ce qui implique, comme le souligne Wolfgang Binder, que Hölderlin traduisant Sophocle tel qu'il eût dî apparaître, si, à un moment favorable du monde, l'auto-accomplissement avait été permis aux Grecs, ne fait rien d'autre, quant à la direction, que ce que fait aussi sa poésie ultérieure, qui veut être une poésie «natale», donc hespérique ${ }^{3}$.

C'est en effet ce qu'il explique à son éditeur Wilmans dans sa lettre du 20 septembre 1803: Par conformisme national et par certains défauts dont il a toujours su s'arranger, l'art grec nous est étranger; j'espère en donner au public une idée plus vivante qu'à l'ordinaire en accentuant le caractère oriental qu'il a toujours renié et en rectifiant, quand il y a lieu, ses défauts esthétiques. ${ }^{4}$ Hölderlin se propose d'orientaliser Sophocle, pour corriger son défaut

\footnotetext{
${ }^{1}$ Cf. W. Binder, Hölderlin et Sophocle, p. 265.

${ }^{2}$ In: F. Hölderlin, Euvres, p. 1000.

${ }^{3}$ W. Binder, Hölderlin et Sophocle, p. 267.

${ }^{4}$ In: F. Hölderlin, Cuvres, p. 1011.
} 
artistique, qui est celui précisément d'un excès d'art. Ce qui caractérise donc Hölderlin, par rapport au purisme classique pour lequel le grec ne peut jamais être assez grec, c'est sa volonté d'accentuer dans le grec le non-grec, l'oriental ${ }^{1}$. Ce terme doit être compris comme le nom d'un des extrêmes entre lesquels se déploie le devenir mondial, comme le laisse entendre ce vers de Wie wenn am Feiertage qui dit de la nature qu'elle est plus ancienne que les temps et audessus des dieux du soir et de l'orient. Car comme l'indiquent aussi plusieurs poèmes, où apparaît une référence à l'Orient, aux forêts de l'Indus (L'aigle, Germanie, L'Ister), aux cités de l'Euphrate et aux rues de Palmyre (Patmos), à l'Asie et à l'est ('A la source du Danube), der Orient, l'Orient, signifie la patrie d'origine du dionysien, c'est-à-dire de l'enthousiasme extatique, comme le dit Hölderlin dans Dichterberuf [Vocation de poète], le poème qu'il écrit au tout début du XIX ${ }^{\mathrm{e}}$ siècle:

Les bords du Gange ont du dieu de la joie entendu

Le triomphe, alors qu'arrivant de l'Indus et

Le monde, le jeune Bacchus avec le vin

conquérant

Sacré tirait les peuples du sommeil. ${ }^{2}$

Oriental signifie donc ici: plus originel, plus libre, plus étranger, non classique, non conventionnel, immédiat, dionysien. Il faut cependant noter que chez Hölderlin la dualité n'est pas, comme chez Nietzsche, celle de Dionysos et d'Apollon, le dionysiaque étant le principe oriental de l'illimité et l'apollinien celui grec de la forme et de la limite. Nietzsche oppose ces deux principes dans La Naissance de la tragédie comme l'art musical à l'art plastique, l'ivresse au rêve, et montre que ce qui différencie les Grecs des Barbares orientaux, c'est précisément la réconciliation de l'orgiaque et de l'organique dans la tragédie où le chœur est l'élément orgiaque et musical, et l'action de personnages le rêve apollinien qui donne forme et limite dans l'élément épique à la vision dionysiaque du chœur. Nietzsche, lui aussi, définit Homère comme l'artiste du rêve qui s'abandonne totalement à la beauté de l'apparence et de la forme, mais il voit en lui la victoire totale de l'illusion apollinienne contre Dionysos alors que Hölderlin y voit celle de la sobriété junonienne contre Apollon. Car Apollon, le dieu solaire, est pour Hölderlin non pas le principe de l'art, mais au contraire celui du feu céleste. Que le dionysiaque nietzschéen corresponde au feu apollinien s'explique sans doute par la double nature d'Apollon, à la fois le plus grec des dieux grecs et pourtant lui aussi d'origine étrangère, asiatique ou nordique, à la fois dieu de l'art, ayant comme attribut la lyre, mais aussi le dieu porteur de l'arc qui menace de loin, envoie la mort et communique l'avenir par oracles, par l'intermédiaire de la Pythie à Delphes.

Que faut-il donc conclure de cet étonnant projet de traduction orientalisante des tragédies de Sophocle? Car il s'agit, il faut le souligner, d'un triple projet: celui d'une transcription d'une langue dans une autre, du grec en allemand; mais aussi d'une transposition de l'original dans un état d'accom-

\footnotetext{
${ }^{1}$ Voir à ce sujet F. Dastur, L'orientalisation de la Grèce.

${ }^{2}$ In: F. Hölderlin, Euvres, p. 778.
} 
plissement qu'il a manqué en faisant ressortir l'oriental sous le grec; enfin d'un accomplissement de l'hespérique lui-même, puisque l'oriental constitue sa tendance culturelle. Ce qui ne signifie pour Hölderlin ni transposer le grec en allemand, ce qui ne serait plus du grec, ni calquer l'allemand sur le grec, ce qui serait encore du grec, mais illisible pour nous, mais corriger l'excès d'art qui a conduit la Grèce à sa perte en faisant apparaître sa nature orientale, c'est-à-dire en fin de compte traduire le grec en grec en le faisant passer dans une autre langue et en accomplissant ainsi ce qu'il n'a pu mener à bien par lui-même. Ce qui se révèle dans une telle tra-duction, c'est, comme le souligne Philippe Lacoue-Labarthe, que la Grèce, 'comme telle, la Grèce' elle-même, 'n'existe

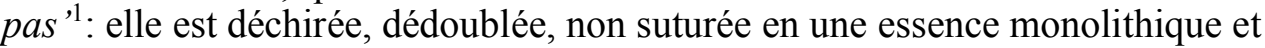
stable, ce qu'il faudrait sans doute dire aussi de tout peuple. Ce que nous connaissons d'elle, le naïf, la sobriété junonienne, la clarté homérique, l'athlétisme de la forme, est un acquis destiné à juguler la puissance élémentaire de la tendance native grecque à transgresser la finitude, ce principe oriental du feu du ciel qui l'empêche de coïncider avec elle-même, et c'est donc, paradoxalement, cette impropriété qui constitue justement ce propre qu'il s'agit, pour les Modernes, d'apprendre, comme toujours, à l'étranger.

\section{Bibliographie}

Alleman B., Hölderlin entre les Anciens et les Modernes in: Hölderlin, (éd.) J.F. Courtine, L'Herne, Paris 1989, pp. 297-321.

Binder W., Hölderlin et Sophocle in: Hölderlin, (éd.) J.-F. Courtine, L'Herne, Paris 1989, pp. 263-276.

Butler E. M., The Tyranny of Grece over Germany. A Study of the Influence Exercised by Greek Art and Poetry over the Great German Writers of the Eighteenth, Nineteenth and Twentieth Centuries, Cambridge University Press, Cambridge 1935 [repr. Beacon Press, Boston 1958].

Chytry J., The Aesthetic State: A Quest in Modern German Thought, University of California Press, Berkeley \& Los Angeles 1989.

Dastur F., Roman et philosophie: l'Hypérion de Hölderlin in: Constitution du champ littéraire. Limites, Intersections, déplacements, (éd.) P. Chiron \& F. Claudon, Cahiers de Philosophie de l'Université Paris 12 Val de Marne 5, 2008, pp. 293-306.

Dastur F., L'orientalisation de la Grèce in: Hölderlin. Le retournement natal, Les Belles Lettres, Paris 2013, pp. 163-187.

Dastur F., Leçons sur la genèse de la pensée dialectique. Schelling, Hölderlin, Hegel, Ellipses, Paris 2016.

Dastur F., Retrait des dieux et modernité selon Novalis et Hölderlin in: Les Études philosophiques 161, 1/2016, pp. 31-44.

Escoubas E., L'Athènes du Nord: Winckelmann à Dresde in: Kairos. La Grèce au miroir de l'Allemagne $\mathrm{n}^{\circ} 16$, Université du Mirail, Toulouse 2000, pp. $13-26$.

\footnotetext{
${ }^{1} \mathrm{Ph}$. Lacoue-Labarthe, Hölderlin et les Grecs, p. 78.
} 
Hegel G. W. F., Fragments de la période de Berne [1793-1796], trad. R. Legros \& F. Verstraten, Vrin, Paris 1987.

Hegel G. W. F., La positivité de la religion chrétienne, trad. G. Planty-Bonjour \& al., PUF, Paris 1983.

Heidegger M., Nietzsche I, trad. P. Klossowski, Gallimard, Paris 1971.

Hölderlin F., Euvres, (éd.) Ph. Jacottet, Gallimard, Paris 1967.

Hölderlin F., Remarques sur Edipe, Remarques sur Antigone, trad. F. Fédier, Union générale d'éditions, Paris 1965.

Janicaud D., Hegel et le destin de la Grèce, Vrin, Paris 1975.

Kant I., Conjectures sur les débuts de l'histoire humaine [1786] in: I. Kant, La philosophie de l'histoire, trad. S. Piobetta, Aubier Montaigne, Paris 1947, pp. 151-172.

Kant I., La philosophie de l'histoire, trad. S. Piobetta, Aubier Montaigne, Paris 1947.

Lacoue-Labarthe Ph., Hölderlin et les Grecs in: L'imitation des Modernes, Galilée, Paris 1986, pp. 71-84.

Mann Th., Gesammelte Werke in dreizehn Bänden, (éd.) H. Bürgin \& P. de Mendelssohn, Fischer, Frankfurt am Main 1974.

Nietzsche F., Crépuscules des Idoles, trad. J.-Cl. Hemery, Gallimard, Paris 1974.

Nietzsche F., Introduction aux études de philologie classique (cours de l'été 1871), trad. F. Dastur \& M. Haar, Encre Marine, La Versanne 1994.

Nietzsche F., La naissance de la tragédie, trad. G. Bianquis, Gallimard, Paris 1949.

Querelle autour de "La naissance de la tragédie", (éd.) M. Dixsaut, Vrin, Paris 1995.

Schiller von F., Poèmes Philosophiques, trad. R. d'Harcourt, Aubier, Paris 1944.

Schiller von F., Poésie naïve et poésie sentimentale, trad. R. Leroux, Aubier, Paris 1947.

Schlegel A., Fragments de l'Athenaeum in: L'absolu littéraire. Théorie de la littérature du romantisme allemand, trad. Ph. Lacoue-Labarthe, J.-L. Nancy \& A.-M. Lang, Seuil, Paris 1978, pp. 98-178.

Schlegel F., Idées in: L'absolu littéraire. Théorie de la littérature du romantisme allemand, trad. Ph. Lacoue-Laberthe, J.-L. Nancy \& A.-M. Lang, Seuil, Paris 1978, pp. 206-223.

Taminiaux J., La nostalgie de la Grèce à l'aube de l'Allemagne classique in: Turm-Vorträge, 1987-88, Hölderlin und die Griechen, Hölderlinturm, Tübingen 1988, pp. 27-43.

Taminiaux J., La nostalgie de la Grèce à l'aube de l'idéalisme allemand. Kant et les Grecs dans l'itinéraire de Schiller, de Hölderlin et de Hegel, Nijhoff, La Haye 1967.

Warminski A., Heidegger en France, trad. F. Dastur in: Hölderlin, (éd.) J.-F. Courtine, L'Herne, Paris 1989, pp. 242-260.

Winckelmann J. J., Réflexions sur l'imitation des ouvres grecques en peinture et en sculpture, trad. L. Mis, Aubier Montaigne, Paris 1954. 